\title{
First Use of Mini Gamma Cameras for Intra-operative Robotic SPECT Reconstruction
}

\author{
Philipp Matthies ${ }^{1}$, Kanishka Sharma ${ }^{1}$, Aslı Okur ${ }^{1,2}$, José Gardiazabal ${ }^{1,2}$, \\ Jakob Vogel ${ }^{1}$, Tobias Lasser ${ }^{1,3}$, and Nassir Navab ${ }^{1}$ \\ 1 Computer Aided Medical Procedures (CAMP), \\ Technische Universität München, Germany \\ 2 Department of Nuclear Medicine, Klinikum Rechts der Isar, \\ Technische Universität München, Germany \\ 3 Institute of Biomathematics and Biometry, Helmholtz Zentrum München, Germany
}

\begin{abstract}
Different types of nuclear imaging systems have been used in the past, starting with pre-operative gantry-based SPECT systems and gamma cameras for $2 \mathrm{D}$ imaging of radioactive distributions. The main applications are concentrated on diagnostic imaging, since traditional SPECT systems and gamma cameras are bulky and heavy. With the development of compact gamma cameras with good resolution and high sensitivity, it is now possible to use them without a fixed imaging gantry. Mounting the camera onto a robot arm solves the weight issue, while also providing a highly repeatable and reliable acquisition platform. In this work we introduce a novel robotic setup performing scans with a mini gamma camera, along with the required calibration steps, and show the first SPECT reconstructions. The results are extremely promising, both in terms of image quality as well as reproducibility. In our experiments, the novel setup outperformed a commercial fhSPECT system, reaching accuracies comparable to state-of-the-art SPECT systems.
\end{abstract}

\section{Introduction}

During the last years, evolving technology has enabled to move further towards intra-operative imaging. This is of tremendous interest, as pre-operative datasets can only be used to a limited extent during surgery. The reason are non-linear discrepancies between the older dataset and the actual situation, as caused for instance by a different patient position or organ deformations. Deformable registration methods exist, but their behavior is usually not sufficiently well-determined to be clinically applicable. Many intra-operative imaging modalities are now natural components of OR equipment, such as Ultrasound or X-ray C-Arm.

A rather recent example is freehand SPECT (fhSPECT) [1, 2] which aims at providing intra-operative nuclear imaging. In SPECT, a radioactive tracer will (ideally) concentrate within a certain targeted anatomical structure, and radioactivity counts acquired from known poses with intersecting perspectives can be used to tomographically reconstruct this distribution. Such information can, when provided in real time, be used for navigation purposes, or to check the 
success of an ongoing procedure. Several groups report on the use of fhSPECT in different surgical procedures [3 $[5]$.

The fhSPECT system basically consists of a hand-held gamma probe (containing one detector, like a Geiger counter) yielding activity counts, and an optical tracking system for locating the former. The surgeon will move the probe over the surface of the region of interest and collect pose/activity pairs along the way. The major difference between a diagnostic SPECT system and fhSPECT is obviously the number and spatial distribution of measurements. While the gantry of a stationary scanner provides statistical significance and good coverage by design, the performance of fhSPECT depends strongly on the abilities of the human operator doing the scan. An inexperienced user will most likely collect biased input data, for instance by leaving out important probe poses or by aiming the probe towards an expected hotspot, thus leaving out important zero-measurements required to carve away regions of no activity. In order to improve the situation, several directions can be taken. One option is to replace the gamma probe, known to have problems in regions with higher background or overlapping activities, with a gamma camera [6], thus obtaining more readings and statistically more meaningful measurements at the same time. Providing measurements on a pixel grid, the device was very bulky while requiring very long acquisition times of $10[\mathrm{~min}]$ and more, and was therefore not suited for intra-operative use. Today, portable mini gamma cameras are available, which weigh less than $1[\mathrm{~kg}]$, while offering good resolution and sensitivity.

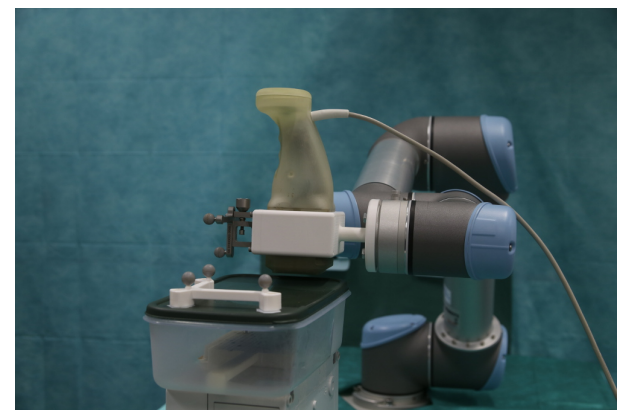

(a) Setup with robot arm

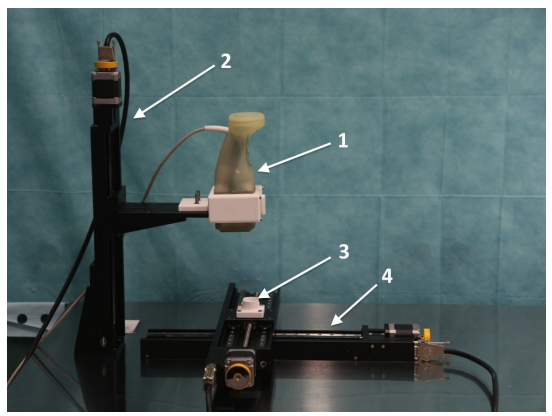

(b) Setup for model measurement

Fig. 1. (1a) Setup with gamma camera attached to robot arm. (1b) Positioning table with gamma camera (1) attached to acquire lookup tables. Custom made parts are used to align the mini gamma camera with the positioning table axes (2 and 4$)$ and to guarantee alignment of radioactive source (3) in front of the detector.

Another important component is the use of a robot arm to guide the imaging sensor, making the scanning process and the image quality reproducible and reliable as it is still not practical to perform hand-held reconstructions on a regular basis. Several classical imaging modalities have recently been combined with robot technology such as C-arm X-ray [7] and laparoscopic ultrasound [8]. 
Bowsher et al. proposed a robotic multi-pinhole SPECT system [9], however only computer simulations were presented. Also for fhSPECT, first steps towards robotic guidance have been taken [10, 11]. For the gamma camera, due to its intrinsic weight and the need of holding it steadily to properly identify the origin of the gamma emission, a robot arm is a natural choice. This, plus the advantage of fast and precise mechanical tracking, provides a perfect match for the gamma camera setup. Potential clinical applications of the robotic mini gamma camera include sentinel lymph node biopsies for breast or head and neck cancer as with fhSPECT, however with faster and more accurate reconstructions allowing to provide better images in more complicated cases such as cervix cancer.

\section{Materials and Methods}

\subsection{Hardware Setup}

The setup consists of a mini gamma camera (Crystal Imager, Crystal Photonics, Germany), mounted on a robot arm (UR5, Universal Robots, Denmark) using a custom-made holder, see Fig. 1a. An optical tracking system (Polaris Vicra, Northern Digital Inc., Canada) was used to track the phantom.

The mini gamma camera comprises of a $4 \times 4\left[\mathrm{~cm}^{2}\right]$ CdZnTe crystal which has $16 \times 16$ pixels. The collimator is made of lead and tungsten and measures $11.15[\mathrm{~mm}]$ in length. It has 256 square holes with sizes of $2.16 \times 2.16\left[\mathrm{~mm}^{2}\right]$ each.

To measure an approximate gamma camera model, the camera was mounted to a precision positioning system (OWIS, Germany), see Fig. $1 \mathrm{~b}$, This positioning system was preferred for its very low repeatability error $(<15[\mu \mathrm{m}])$.

For comparison, a fhSPECT system (declipseSPECT, SurgicEye, Germany) with a single pixel detector (HiSens, Crystal Photonics, Germany) was employed.

\subsection{Gamma Camera Modeling}

For SPECT reconstruction, a model of the gamma camera response is needed. We measure an approximation using a ${ }^{57}$ Co calibration source $(0.97[M B q]$ at time of acquisition) and a three axes precision positioning system. As illustrated in Fig. 1b the mini gamma camera is mounted on a holder (1) that can move along the z-axis (2). The calibration source is mounted on a holder (3) that can be moved in $\mathrm{x}$ - and $\mathrm{y}$-direction (4).

The source is scanned in a $150 \times 150 \times 150\left[\mathrm{~mm}^{3}\right]$ grid with step size $5[\mathrm{~mm}]$ over the centered source. A volume of $50 \times 50 \times 50\left[\mathrm{~mm}^{3}\right]$ directly centered in front of the camera is rastered with a finer step size of $1[\mathrm{~mm}]$. At each grid position, an image is acquired with $7[s]$ exposure time.

The resulting measurements were corrected by a homogeneity correction factor and an energy window of $\pm 10 \%$ was applied after energy calibration had been performed. For each detector pixel $k$ the measurements are then stored in a lookup table $\ell^{k}$ for later use in the reconstruction method. The area covered by the lookup table is illustrated in Fig. 2a, Fig. 2b shows a $3 \mathrm{D}$ rendering of the values of the lookup table for one example detector pixel. 


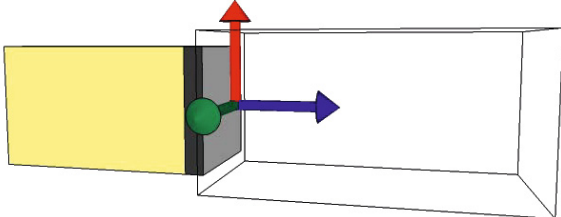

(a) Lookup table illustration

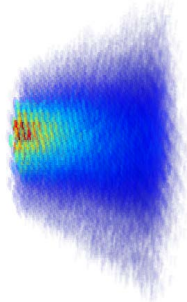

(b) Measured lookup table

Fig. 2. (2a) Drawing of gamma camera with volume of lookup table in front of it illustrating the dimensions and orientations of the reference coordinate system. (2b) Graph showing an exemplary lookup table for an arbitrary pixel. The gamma camera is centered on the left face of the volume pointing to the right. Contributions of the different voxels in the volume in front of the camera to the specific pixel are shown. Red indicates a high contribution, blue a low one.

\subsection{Coordinate Systems Calibration}

Corresponding activity measurements and camera poses are the key to tomographic reconstruction. The optical tracking system provides pose information for a tracking target cam mounted to the camera, but in order to relate measurements via the lookup tables to the world system, the world locations of the detector pixels are required.

First, we define a detector coordinate system compatible to the lookup table (Fig. 2a ), centered on the detector face, $x$ - and $y$ - axes aligned with the pixel grid and $z$ pointing away from the camera towards the measurement region. In order to obtain the respective transformation ${ }^{\text {cam }} \mathbf{T}_{\text {detector }}$, we first compute the detector coordinate system in absolute terms and derive the relative transformation afterwards.

Consequently, we measure the four corner points of the detector face using a calibrated optical pointer (Fig. [3), yielding $\mathbf{c}_{ \pm x, \pm y} \in \mathbb{R}^{3}$. From these four points, we compute the mean and obtain the center/base point $\mathbf{0}_{\text {detector }}=\mu\left(\mathbf{c}_{ \pm x, \pm y}\right)$ where $\mu$ denotes a function yielding the $n$-dimensional arithmetic mean. Using the averaged delta vectors $\mathbf{v}_{x}^{\prime}=\mu\left(\mathbf{c}_{+x, \pm y}-\mathbf{c}_{-x, \pm y}\right)$ and $\mathbf{v}_{y}^{\prime}=\mu\left(\mathbf{c}_{ \pm x,+y}-\mathbf{c}_{ \pm x,-y}\right)$, we then compute the $x$ - and $y$-axes as $\mathbf{v}_{x}=\mathbf{v}_{x} /\left\|\mathbf{v}_{x}\right\|$ and $\mathbf{v}_{y}=\mathbf{v}_{y} /\left\|\mathbf{v}_{y}\right\|$. The $z$-axis is finally computed via the cross product as $\mathbf{v}_{z}=\mathbf{v}_{x} \times \mathbf{v}_{y}$. From the base point and the three axes we obtain the detector coordinate system, and compute the incremental transformation ${ }^{\text {cam }} \mathbf{T}_{\text {detector }}$.

\subsection{Reconstruction Method}

Let $f: V \rightarrow \mathbb{R}$ denote the activity to be reconstructed in the volume of interest $V \subset \mathbb{R}^{3}$. Given a set of $n$ basis functions $b_{i}: V \rightarrow \mathbb{R}$, we discretize $f \approx \sum_{i=1}^{n} x_{i} b_{i}$ 


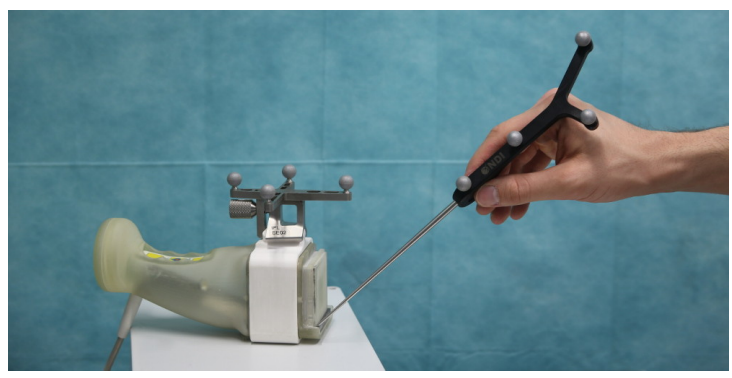

Fig. 3. An optical pointer was used to correlate the detector front face with the optical tracking target attached to the mini gamma camera

with a coefficient vector $\mathbf{x}=\left(x_{i}\right) \in \mathbb{R}^{n}$. In this work, the $b_{i}$ are voxel basis functions, chosen in a $56 \times 50 \times 22$ grid with voxel size $2.5 \times 2.5 \times 2.5\left[\mathrm{~mm}^{3}\right]$.

The measured lookup tables $\ell^{k}$ at each gamma camera pixel $k$ are then used to map each voxel $b_{i}$ to a camera measurement $m_{j}^{k}$ at position $p_{j}$ via nearest neighbor interpolation, yielding a linear measurement model $\mathcal{M}_{j}^{k}\left(b_{i}\right)$. For each camera position $p_{j}$ and camera pixel $k$ we then have $\mathcal{M}_{j}^{k}(f)=m_{j}^{k}$ using the model $\mathcal{M}_{j}^{k}$, with $\mathbf{m}=\left(m_{j}^{k}\right) \in \mathbb{R}^{l}$ denoting the camera readouts. Together, this allows to formulate the discretized reconstruction problem as

$$
m_{j}^{k}=\mathcal{M}_{j}^{k}(f) \approx \sum_{i=1}^{n} x_{i} \mathcal{M}_{j}^{k}\left(b_{i}\right) .
$$

Using the short notation $a_{j i}^{k}=\mathcal{M}_{j}^{k}\left(b_{i}\right)$ we define the system matrix $\mathbf{A}=\left(a_{j i}^{k}\right)$. Now the reconstruction problem can be formulated as solving an inconsistent system of linear equations $\mathbf{A x}=\mathbf{m}$ [10]. After removal of columns and rows being entirely zero from $A$ to avoid singularities, we solve the least squares problem $\min _{\mathbf{x}}\|\mathbf{A x}-\mathbf{m}\|^{2}$ using a standard maximum likelihood expectation maximization (MLEM) algorithm with 20 iterations. Finally, the computed approximation to $\mathbf{x}$ allows computation of the desired activity map $f$.

\subsection{Experimental Procedure}

A phantom was made mimicking a sentinel lymph node scenario. Three hollow spheres $(2 \times 0.2[\mathrm{ml}]$ and $0.1 \mathrm{ml})$ were screwed into a plastic box and an optical tracking target was attached on the lid, as shown in Fig 4a, The spheres were filled with ${ }^{99 m} \mathrm{Tc}(650[\mathrm{kBq}]$ in sphere $(1), 850[\mathrm{kBq}]$ in $(2)$ and $500[\mathrm{kBq}]$ in (3), cf. Fig. 4b). Five robotic gamma camera scans were performed with an acquisition time of $60[s]$ for each scan from the directions shown in Fig. 4b. The robot was moving to 6 previously defined positions on 3 orthogonal planes and takes gamma camera acquisitions of $10[s]$. In each scan the angle, position and orientation of the gamma camera was slightly changed. For comparison we also scanned 5 times with the fhSPECT system with a duration of $60[s]$ and comparable scanning directions. 


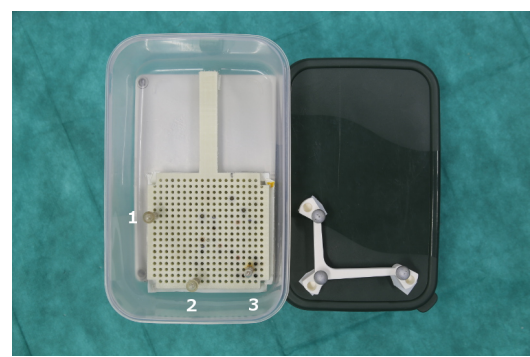

(a) Phantom from top

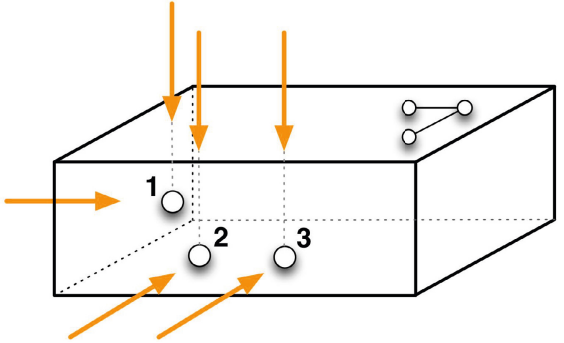

(b) Scanning directions

Fig. 4. (4a) Phantom from top view with the three spheres filled with radioactive ${ }^{99 m}$ Tc solution, the lid with the optical tracking target attached to it lies beneath the box to reveal the view inside. (4b) Scanning directions of the robot moving the mini gamma camera. All the spheres were scanned from one side and from the top. The freehand gamma probe scans covered the same three sides homogeneously.

\section{$3 \quad$ Evaluation and Results}

Five gamma camera scans were performed and compared to a CT image of the phantom as ground truth with the ${ }^{99 m}$ Tc filled spheres clearly visible. The reconstructions yielded mean distances to their respective ground truth of $4.3[\mathrm{~mm}]$ $(\sigma=0.3[\mathrm{~mm}]$, sphere 1$), 4.0[\mathrm{~mm}](\sigma=0.9[\mathrm{~mm}]$, sphere 2) and $5.6[\mathrm{~mm}]$ $(\sigma=3.4[\mathrm{~mm}]$, sphere 3$)$. In all five scans the three spheres could be reconstructed successfully, only in one reconstruction a larger artifact close to one sphere showed up, in two other scans one small artifact was visible close to the correctly reconstructed spheres and to the border of the volume. These artifacts result from the nature of the used model as the lookup tables contain the largest values close to the detector and when the volume of interest is very close to the detector positions, the border regions are intensified.

The fhSPECT reconstructions were then compared to the ground truth as well. Only in one case all three spheres could be reconstructed, although combined with additional artifacts (cp. arrows in Fig.5b). In the remaining four scans 2 of the 3 hot spots could be detected without major artifacts in the images. The mean distance of the reconstructed spheres to their respective ground truth positions were $20.4[\mathrm{~mm}](\sigma=3.1[\mathrm{~mm}])$, sphere 1$)$ and $16.8[\mathrm{~mm}](\sigma=2.2[\mathrm{~mm}])$, sphere 2). Sphere 3 could only be reconstructed in one scan with a distance of $27.2[\mathrm{~mm}]$ to its ground truth position. This resulted with the tradeoff of large artifacts in the image. However, total counts acquired with the mini gamma camera were more than 10 times higher and the fhSPECT scanning time is considered short compared to clinical practice.

Fig. 5a shows a reconstruction performed using a gamma camera mounted to a robot arm and scanning the phantom from 6 different positions for $10[s]$ each. Fig. 5b shows a reconstruction performed with fhSPECT device and a gamma probe after scanning the volume of interest for $60[s]$ from three orthogonal sides. 


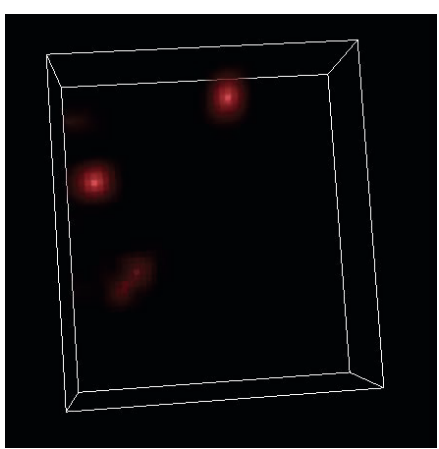

(a) Camera reconstruction

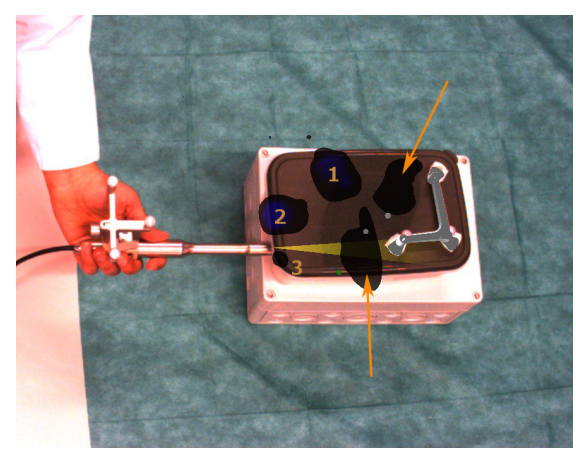

(b) Probe reconstruction

Fig. 5. Reconstruction performed using a gamma camera mounted to a robot arm (5a) and with fhSPECT device and a gamma probe (5b). Arrows indicate artifacts.

\section{Discussion and Conclusion}

The upgrade from a single gamma detector to a compact gamma camera for 3D freehand scans has a huge impact on the quality of the tomographic reconstructions achieved by comparable computation times $(<1[\mathrm{~min}])$. The fact that camera data enables reconstructing hot-spots with lower activity, the novel system allows faster acquisitions and lower tracer doses, thus limiting the exposure, and therefore the risk associated with the procedure.

This is, of course, not without trade-offs, most importantly the weight of the camera. This can be solved by using a robot arm like it is proposed in this paper, or by a mechanical construction to support it.

The ability of reconstructing images with an accuracy close to a diagnostic SPECT machine, coupled with the low presence of artifacts and the increased precision of the hot-spot relocation are clear benefits for the medical workflow. However, the purpose of intra-operative SPECT is guidance and not diagnosis.

The use of a robot arm enables the use of optimized acquisition trajectories, or feedback loops, since it is also possible to use the current acquisitions to perform on-the-fly adaptive trajectories. This combination can lead to even shorter acquisition times, while keeping the quality constant. The robot used for the experiments is not a medical device, and it does not fulfill the minimum safety requirements for its use in the operation room [12]. However, it is possible to use for phantom studies and ex-vivo experiments.

Future applications could include radioembolization therapy [13] using ${ }^{99 m} \mathrm{Tc}-$ MAA in concordance with intra-operative CT images to better localize any suspicious foci of activity.

Acknowledgements. This work was partially funded by the DFG SFB 824 and the DFG cluster of excellence MAP. 


\section{References}

1. Wendler, T., Hartl, A., Lasser, T., Traub, J., Daghighian, F., Ziegler, S.I., Navab, N.: Towards Intra-operative 3D Nuclear Imaging: Reconstruction of 3D Radioactive Distributions Using Tracked Gamma Probes. In: Ayache, N., Ourselin, S., Maeder, A. (eds.) MICCAI 2007, Part II. LNCS, vol. 4792, pp. 909-917. Springer, Heidelberg (2007)

2. Wendler, T., Herrmann, K., Schnelzer, A., Lasser, T., Traub, J., Kutter, O., Ehlerding, A., Scheidhauer, K., Schuster, T., Kiechle, M., Schwaiger, M., Navab, N., Ziegler, S.I., Buck, A.K.: First demonstration of 3-D lymphatic mapping in breast cancer using freehand SPECT. Eur. J. Nucl. Med. 37(8), 1452-1461 (2010)

3. Schnelzer, A., Ehlerding, A., Blümel, C., Okur, A., Scheidhauer, K., Paepke, S., Kiechle, M.: Showcase of intraoperative 3D imaging of the sentinel lymph node in a breast cancer patient using the new freehand SPECT technology. Breast Care 7(6), 484-486 (2012)

4. Heuveling, D.A., Karagozoglu, K.H., van Schie, A., van Weert, S., van Lingen, A., de Bree, R.: Sentinel node biopsy using 3D lymphatic mapping by freehand SPECT in early stage oral cancer: a new technique. Clin. Otolaryngol. 37(1), 89-90 (2012)

5. Naji, S., Tadros, A., Traub, J., Healy, C.: Case report: Improving the speed and accuracy of melanoma sentinel node biopsy with 3D intra-operative imaging. J. Plast. Reconstr. Aes. 64(12), 1712-1715 (2011)

6. Weinberg, I., Zawarzin, V., Pani, R., De Vincentes, G.: Implementing reconstruction with hand-held gamma cameras. In: IEEE Nuclear Science Symposium Conference Record, vol. 104, pp. 21/101-21/104 (2000)

7. Ganguly, A., Fieselmann, A., Marks, M., Rosenberg, J., Boese, J., Deuerling-Zheng, Y., Straka, M., Zaharchuck, G., Bammer, R., Fahrig, R.: Cerebral CT Perfusion Using an Interventional C-Arm Imaging System: Cerebral Blood Flow Measurements. Am. J. Neuroradiol. 32, 1525-1531 (2011)

8. Schneider, C., Guerrero, J., Nguan, C., Rohling, R., Salcudean, S.: Intra-operative "Pick-Up" Ultrasound for Robot Assisted Surgery with Vessel Extraction and Registration: A Feasibility Study. In: Taylor, R.H., Yang, G.-Z. (eds.) IPCAI 2011. LNCS, vol. 6689, pp. 122-132. Springer, Heidelberg (2011)

9. Bowsher, J., Yan, S., Roper, J., Giles, W., Yin, F.: TU-A-BRA-01: a robotic multipinhole SPECT system for onboard and other region-of-interest imaging, vol. 39, pp. 3887-3888. AAPM (2012)

10. Vogel, J., Reichl, T., Gardiazabal, J., Navab, N., Lasser, T.: Optimization of acquisition geometry for intra-operative tomographic imaging. In: Ayache, N., Delingette, H., Golland, P., Mori, K. (eds.) MICCAI 2012, Part III. LNCS, vol. 7512, pp. 42-49. Springer, Heidelberg (2012)

11. Gardiazabal, J., Reichl, T., Okur, A., Lasser, T., Navab, N.: First flexible robotic intra-operative nuclear imaging for image-guided surgery. In: Barratt, D., Cotin, S., Fichtinger, G., Jannin, P., Navab, N. (eds.) IPCAI 2013. LNCS, vol. 7915, pp. 81-90. Springer, Heidelberg (2013)

12. Taylor, R., Paul, H., Kazanzides, P., Mittelstadt, B., Hanson, W., Zuhars, J., Williamson, B., Musits, B., Glassman, E., Bargar, W.: Taming the bull: safety in a precise surgical robot. In: Fifth International Conference on Advanced Robotics (ICAR), vol. 1, pp. 865-870 (1991)

13. Peynircioğlu, B., Çil, B., Bozkurt, F., Aydemir, E., Uğur, O., Balkancı, F.: Radioembolization for the treatment of unresectable liver cancer: initial experience at a single center. Diagn. Interv. Radiol. 16(1), 70-78 (2010) 